University of Nebraska - Lincoln

DigitalCommons@University of Nebraska - Lincoln

8-2013

\title{
Disturbance Frequency and Vertical Distribution of Seeds Affect Long-Term Population Dynamics: A Mechanistic Seed Bank Model
}

\author{
Eric A. Eager \\ University of Wisconsin - La Crosse, eeager@uwlax.edu \\ Chirakkal V. Haridas \\ University of Nebraska-Lincoln \\ Diana Pilson \\ University of Nebraska-Lincoln, dpilson1@unl.edu \\ Richard Rebarber \\ University of Nebraska-Lincoln, rrebarber1@unl.edu \\ Brigitte Tenhumberg \\ University of Nebraska - Lincoln, btenhumberg2@unl.edu
}

Follow this and additional works at: https://digitalcommons.unl.edu/bioscifacpub

Part of the Biology Commons

Eager, Eric A.; Haridas, Chirakkal V.; Pilson, Diana; Rebarber, Richard; and Tenhumberg, Brigitte, "Disturbance Frequency and Vertical Distribution of Seeds Affect Long-Term Population Dynamics: A Mechanistic Seed Bank Model" (2013). Faculty Publications in the Biological Sciences. 308.

https://digitalcommons.unl.edu/bioscifacpub/308

This Article is brought to you for free and open access by the Papers in the Biological Sciences at DigitalCommons@University of Nebraska - Lincoln. It has been accepted for inclusion in Faculty Publications in the Biological Sciences by an authorized administrator of DigitalCommons@University of Nebraska - Lincoln. 


\title{
Disturbance Frequency and Vertical Distribution of Seeds Affect Long-Term Population Dynamics: A Mechanistic Seed Bank Model
}

\author{
Eric Alan Eager, ${ }^{1, \star}$ Chirakkal V. Haridas, ${ }^{2}$ Diana Pilson, ${ }^{2}$ Richard Rebarber, ${ }^{3}$ and \\ Brigitte Tenhumberg ${ }^{2,3}$
}

\begin{abstract}
1. Mathematics Department, University of Wisconsin, La Crosse, Wisconsin 54601; 2. School of Biological Sciences, University of Nebraska, Lincoln, Nebraska 68588; 3. Department of Mathematics, University of Nebraska, Lincoln, Nebraska 68588
\end{abstract}

Submitted October 8, 2012; Accepted March 18, 2013; Electronically published June 21, 2013

Online enhancements: supplementary figures and R code.

\begin{abstract}
AвsтRACт: Seed banks are critically important for disturbance specialist plants because seeds of these species germinate only in disturbed soil. Disturbance and seed depth affect the survival and germination probability of seeds in the seed bank, which in turn affect population dynamics. We develop a density-dependent stochastic integral projection model to evaluate the effect of stochastic soil disturbances on plant population dynamics with an emphasis on mimicking how disturbances vertically redistribute seeds within the seed bank. We perform a simulation analysis of the effect of the frequency and mean depth of disturbances on the population's quasi-extinction probability, as well as the long-term mean and variance of the total density of seeds in the seed bank. We show that increasing the frequency of disturbances increases the long-term viability of the population, but the relationship between the mean depth of disturbance and the long-term viability of the population are not necessarily monotonic for all parameter combinations. Specifically, an increase in the probability of disturbance increases the long-term viability of the total seed bank population. However, if the probability of disturbance is too low, a shallower mean depth of disturbance can increase long-term viability, a relationship that switches as the probability of disturbance increases. However, a shallow disturbance depth is beneficial only in scenarios with low survival in the seed bank.
\end{abstract}

Keywords: disturbance specialist, annual plant, seed bank, stochastic integral projection model, density dependence, Monte Carlo simulation.

\section{Introduction}

In many plant species dormant seeds persist in the soil from one to several years (MacDonald and Watkinson 1981; Roberts 1981; Doyle et al. 1986; McGraw 1986; Maxwell et al. 1988; Venable 1989; Doyle 1991; Kalisz and

\footnotetext{
* Corresponding author; e-mail: eeager@uwlax.edu.
}

Am. Nat. 2013. Vol. 182, pp. 180-190. (C) 2013 by The University of Chicago. 0003-0147/2013/18202-54165\$15.00. All rights reserved. DOI: $10.1086 / 670987$
McPeek 1992; Jordan et al. 1995; Gonzalez-Andujar 1997; Edelstein-Keshet 2005; Fenner and Thompson 2005; Pekrun et al. 2005; Garnier et al. 2006; Sester et al. 2007; Colbach et al. 2008). By allowing individuals to disperse through time, these seed banks function to buffer the effects of environmental variation on population size and persistence. Such environmental buffering is particularly important for annual species. Many annual plants are disturbance specialists, germinating only in freshly disturbed soil (Alexander and Schrag 2003). In these species the frequency, intensity, timing, and spatial extent of natural disturbances can greatly influence the probability of germination and survival of seeds in the seed bank (FroudWilliams et al. 1984; Claessen et al. 2005a; Moody-Weis and Alexander 2007; Miller et al. 2011). Disturbances not only create a more favorable environment for germination and recruitment by removing more competitive species, but they also enhance the chance of population persistence by helping seed bank formation (Alexander et al. 2009).

It has long been known that seed survival and germination are functions of seed depth in the seed bank (Moody-Weis and Alexander 2007; and reviewed in Mohler 1993). This knowledge has attracted much attention in agricultural research, and many models have been constructed to explore different plowing regimes to manipulate the distribution of weed seeds in the soil in order to reduce weed population size in agricultural fields (Doyle et al. 1986; McGraw 1986; Maxwell 1988; Mohler 1993; Jordan et al. 1995; Gonzalez-Andujar 1997; Mertens et al. 2002; Pekrun et al. 2005; Garnier and Lecomte 2006; Sester et al. 2007; van den Berg et al. 2010). However, in addition to anthropogenic disturbances, many natural processes move seeds to different soil depths. For example, seeds are moved from the surface to lower soil depth through earthworm cast and mole burial, caching activities of birds, 
rodents, ants, and so on, or seeds may fall down earthworm burrows or cavities left after root decay. Seeds are moved from lower seed depths to the surface through settling of the soil, digging activities by mammals such as badgers, black bears, wild pigs, and so on or falling trees bringing buried seeds up. In this article, we use a stagestructured population model (Easterling et al. 2000; Caswell 2001) to examine the population dynamics of disturbance specialist plants in a natural environment, where disturbances occur in a much more unpredictable fashion than those that occur in agricultural fields. To our knowledge, only two articles (Claessen et al. 2005a, 2005b) have considered disturbances in natural environments that are random in time, but, in contrast to our article, they do not explicitly consider disturbance specialist plants.

In this article, we present a model for disturbance specialist plants in which we assume that germination is possible only in time steps where there is a disturbance. To mimic disturbances in a natural environment we incorporate the characteristics of a disturbance as a stochastic process, where in each time step there is a probability $h$ that the population is disturbed and the distribution of possible disturbance depths is exponentially distributed with mean depth of disturbance $\rho$. Models considering soil disturbances caused by different plowing regimes (Doyle et al. 1986; McGraw 1986; Maxwell 1988; Mohler 1993; Jordan et al. 1995; Gonzalez-Andujar 1997; Mertens et al. 2002; Pekrun et al. 2005; Garnier and Lecomte 2006; Sester et al. 2007; van den Berg et al. 2010) can be thought of as mean-field approximations of models with random disturbances. They consist of deterministic matrices (and thus integral kernels) and represent the average effect of soil disturbances. However, in the case of nonlinear relationship, using averages might be inaccurate because the convex transformation of a mean is less than the mean after convex transformation, and the opposite holds for concave transformations (Jensen's inequality). Thus, the long-term dynamics of mean-field models may not accurately reflect the dynamics of an associated stochastic model and, hence, do not always capture variability in natural systems (Tuljapurkar 1990; Caswell 2001).

\section{The Model}

We model the following sequence of events: disturbance, redistribution of seeds, seed survival, plant recruitment, and production of new seeds. We consider only disturbances which occur after seeds have been dispersed because disturbances before dispersal have a negligible effect on the seed bank (Moody-Weis and Alexander 2007). We model disturbance as a single event in each time step, which can be thought of as an average of the postdispersal disturbances to the population in a given year. The life- history traits of different plants species vary considerably, and as a consequence, the functional forms we use to model survival probability, germination probability, and density dependence do not apply in every case but apply to a large number of disturbance specialist plant species (Chancellor 1964; Froud-Williams et al. 1984; Mohler 1993; Sester 2007; Colbach et al. 2008).

\section{Disturbance and Redistribution of Seeds}

We model disturbances as independent and identically distributed stochastic events influencing the population dynamics each year. The random disturbances are denoted by $\theta(t)$. At each time step $t$ we break $\theta(t)$ into two random variables, $\theta_{1}(t)$ and $\theta_{2}(t)$. Term $\theta_{1}(t)$ is a Bernoulli random variable determining whether or not the population is disturbed, which is equal to unity with the probability of disturbance $h$ and zero with the probability of no disturbance $1-h$. Given that a disturbance occurs, $\theta_{2}(t)$ determines the depth at which the disturbance affects the seeds in the population at time $t$. For example, if $\theta(t)=0.5 D$, then the disturbance uniformly redistributes all seeds above one-half of the maximum depth of the seed bank $([0, D / 2])$ and leaves the rest of the seed bank $([D / 2, D])$ undisturbed. The depth of a disturbance is modeled as a truncated exponential distribution with mean depth of disturbance $\rho$. Thus,

$$
\operatorname{Pr}\left\{\theta \leq r \mid \theta_{1}(t)=1\right\}=\left\{\begin{array}{ll}
1-\exp (-r / \rho) & \text { if } r<D \\
1 & \text { if } r=D
\end{array},\right.
$$

where $\theta_{2}$ is the depth of disturbance. We then define the disturbances $\theta(t)$ with the following equation:

$$
\theta(t)=\theta_{1}(t) \theta_{2}(t)
$$

for every $t=0,1,2, \ldots$ Using this definition, $\theta(t)$ determines both the occurrence and depth of disturbance for each time $t$. We define the disturbance kernel $K$ at time $t$ for the disturbance $\theta(t)$, acting on the population $u$, as

$$
\int_{0}^{D} K(x, y, \theta(t)) u(y) d y=\left\{\begin{array}{ll}
1 / \theta(t) \int_{0}^{\theta(t)} u(y) d y & \text { if } 0 \leq x \leq \theta(t) \\
u(x) & \text { if } \theta(t)<x \leq D
\end{array},\right.
$$

with the convention that the top term on the right-hand side of equation (3) is equal to zero when $\theta(t)=0$. The top term in the right-hand side of equation (3) is modeling the population $u$ being uniformly redistributed within the interval $[0, \theta(t)]$, and the bottom term is the population $u$ being left alone within the interval $[\theta(t), D]$. The dimension of $K$ is (depth) ${ }^{-1}$ for all $\theta(t)$. 


\section{Survival}

Only a fraction of the seeds that do not germinate survive to the next time step. We make the simplifying assumptions that this fraction depends only on the seed's depth $x$ in the seed bank (Mohler 1993), that seeds survive at their lowest rates near the surface of the soil (due largely to seed predation), and that the likelihood of survival increases as seed depth increases. To model survival as a function of depth we use the function

$$
s(x)=s_{0}(1-\exp (-b x)),
$$

where $0<s_{0}<1$ is the maximum survival probability of a seed and $b$ models the incremental gain in survival probability that occurs through an incremental increase in seed depth (Mohler 1993). The function $s$ is dimensionless.

\section{Plant Recruitment}

We assume that germination occurs only in the presence of a disturbance, and thus, the germination probability is a function of $\theta(t)$; that is,

$$
g(x, \theta(t))=\left\{\begin{array}{ll}
g_{p}(x) & \text { if } \theta(t) \neq 0 \\
0 & \text { if } \theta(t)=0
\end{array},\right.
$$

where $g_{p}(x)$ is the probability of a seed of depth $x$ germinating in a given time step, given a disturbance. We assume that the probability of a seed germinating is such that seeds germinate at their highest rate near the surface of the soil, and the likelihood of germination drops as seed depth increases (Chancellor 1964; Sester 2007). To model germination as a function of depth we use the function

$$
g_{p}=g_{0} \exp (-a x)
$$

where $0<g_{0}<1$ is the probability of a seed on the surface of the soil germinating and $a$ models the loss in germination probability that occurs through an incremental increase in seed depth (Mohler 1993). The function $g$ is dimensionless.

If conditions are favorable and the seed bank size is large, it is possible that even disturbance specialist plants experience density dependence in some years (see, e.g., fig. 3 in Alexander and Schrag 2003). Thus, we explicitly included density dependence in our model by assuming that seedling recruitment decreases with seedling density. We assume that seedling recruitment follows a Holling type II functional form (Holling 1959). A derivation of an analogous relationship for a general plant population is available in Eager et al. (2012), which utilizes the idea of competing for a finite number of available microsites. The number of plants $f(x)$ that result from $x$ seeds $(\text { area })^{-1}$ is assumed to be

$$
f(x)=\frac{\alpha x}{\beta+x} .
$$

The parameter $\alpha$ is the maximum number of adult plants that can grow in a given area, with dimension plants $(\text { area })^{-1}$. Term $\beta$ (with dimension seeds $(\text { area })^{-1}$ ) is the half-saturation constant, that is, the density of seedlings that would end up producing half of the maximum population size, $\alpha$. Term $f$ is the only term in the model that is density dependent. The dimension of $f$ is plants $(\text { area })^{-1}$.

\section{Seed Production}

We assume that seed production is not affected by plant density because we show in Eager et al. (2013) that this would have a negligible effect on the equilibrium population, as our model already considers density dependence in the seedling-to-plant transition. In this example, we do not model the size structure of the plants explicitly because we envision annual plants, and the model uses a time step of 1 year. Each plant produces an average number of seeds $c$, which are distributed according to the depth distribution $J$. We assume that $J$ is a truncated exponential distribution with mean $\mu \ll 1$ (Mohler 1993), which ensures that most seeds are set on the surface of the soil. As a consequence, most newly created seeds die if there is no disturbance in the following year. The dimension of $c$ is seeds (plant) ${ }^{-1}$ and $J$ has dimension $(\text { depth })^{-1}$.

\section{Integral Projection Model}

We construct a stochastic integral projection model (IPM; Easterling 2000; Ellner 2006; Ellner and Rees 2007; Coulson 2012) because we want to include seed depth as a continuous stage variable. Other articles have treated seed depth as a discrete variable and constructed matrix models (Doyle et al. 1986; McGraw 1986; Jordan et al. 1995; Gonzalez-Andujar 1997; Claessen et al. 2005a, 2005b; Colbach et al. 2008; Pekrun et al. 2005; Mohler 2006). However, model predictions (i.e. long-term growth rates, sensitivities/elasticities, and transient dynamics) can change as matrix dimensions increase, even when using the same data set (Easterling et al. 2000; Ellner and Guckenheimer 2006; Tenhumberg et al. 2009). By using an IPM we do not have to explore the effect of the number of seed layers on model predictions.

Our IPM simulates how the distribution of seeds in the seed bank and the plant population density of an annual species changes from one year to the next. Let $n(x, t)$ be the density of seeds in the seed bank at time $t$ between depths $x$ and $x+d x$ and $p(t)$ be the density of plants in the population at time $t$, for $t=0,1,2, \ldots$. The dimensions of $n$ and $p$ are seeds $(\text { depth })^{-1}(\text { area })^{-1}$ and plants 
Table 1: Model parameters

\begin{tabular}{lccl}
\hline Parameter & Value & Equation & \multicolumn{1}{c}{ Scenario } \\
\hline Maximum survival $s_{0}$ & .5 & $(4)$ & Low seed survival \\
Maximum survival $s_{0}$ & .95 & $(4)$ & High seed survival \\
Rate of change in survival $b$ & 10 & $(4)$ & Neutral \\
Rate of change in survival $b$ & 5 & $(4)$ & Neutral \\
Maximum germination $g_{0}$ & .95 & $(6)$ & High germination \\
Maximum germination $g_{0}$ & .5 & $(6)$ & Low germination \\
Rate of change in germination $a$ & 10 & $(6)$ & Neutral \\
Rate of change in germination $a$ & 5 & $(6)$ & Neutral \\
Mean depth of dispersal $\mu$ (which appears in $J(x))$ & .02 & $(8)$ & Neutral \\
Holling parameters $\alpha, \beta$ & 40,50 & $(7)$ & Low carrying capacity \\
Holling parameters $\alpha, \beta$ & 400,500 & $(7)$ & High carrying capacity \\
Holling parameters $\alpha, \beta$ & 40,500 & $(7)$ & Low establishment \\
Seed production per plant $c$ & 50 & $(8)$ & Low fecundity \\
Seed production per plant $c$ & 150 & $(8)$ & High fecundity \\
\hline
\end{tabular}

Note: Parameter values for simulations of the model in equation (8).

$(\text { area })^{-1}$, respectively. We assume that all plants in the population behave like the average plant, so $p(t)$ is simply a nonnegative number for all $t$. The model is therefore

$$
\begin{aligned}
n(x, t+1)= & s(x)[1-g(x, \theta(t)] \\
& \times \int_{0}^{D} K(x, y, \theta(t))(n(y, t)+c J(y) p(t)) d y, \\
p(t+1)= & f\left(\int_{0}^{D} g(x, \theta(t))\right. \\
& \left.\times \int_{0}^{D} K(x, y, \theta(t))(n(y, t)+c J(y) p(t)) d y d x\right\},
\end{aligned}
$$

for $t=0,1,2, \ldots$ and $n(x, 0)>0$. The first line in model (8) states that the buried seed distribution at time $t+1$ results from three processes: redistribution by disturbance at time $t$ (from the integral involving the kernel $K$ ), the fraction of seeds that do not germinate at time $t$ (from $1-g$ ), and the fraction of seeds that survive from time $t$ to $t+1$ (from $s$ ). The second line states that the density of plants at time $t+1$ results from three processes: redistribution of seeds by disturbance at time $t$, germination of a fraction of these disturbed seeds at time $t$ (from $g$ ), and the recruitment of the seedlings that come from these germinated seeds (via the function $f$ ).

In this article we use simulations to conjecture that as $t \rightarrow \infty$, the population sequence $\{[n(x, t), p(t)]\}_{t=0}^{\infty}$ converges to a stationary random population $\left[n(x)^{*}, p^{*}\right]^{\mathrm{T}}$, independent of the nonzero initial population $[n(x, 0), p(0)]^{\mathrm{T}}$, which is in agreement with the mathematical results for other similar stochastic models (Hardin et al. 1988; Benaim and Schreiber 2009). Thus, as $t \rightarrow \infty$, we conjecture that the probability distribution of the population converges (and thus the long-term population has a constant mean and variance). We perform Monte Carlo simulations to analyze how the mean and variance of the populations change with changes in the disturbance parameters $h$ (the probability of disturbance) and $\rho$ (the mean depth of disturbance). We also explore the effect of changing fecundity (seeds per plant) $c$, recruitment parameters $\alpha$ and $\beta$ (from eq. [7]), seed survival parameters $s_{0}$ and $b$ (from eq. [4]), and germination parameters $g_{0}$ and $a$ (from eq. [6]).

\section{Model Analysis}

All simulations were done in $\mathrm{R}$ ( $\mathrm{R}$ Core Development Team 2006), using numerical integration techniques explained in Ellner and Rees (2007), with parameter values listed in table 1. All computer programs used in our simulations are provided in a text file in the supplementary material, available online. ${ }^{1}$ In our simulation studies we set the maximum depth $D=1$ so that shallower depths are represented as a proportion of the maximum depth. We considered a range of different ecological scenarios. The values $\alpha=40$ and $\beta=50$ were consistent with figure 3 in Alexander and Schrag (2003) for wild sunflower (Helianthus annuus). Figure S1 (figs. S1-S5 available online) illustrates for one simulation how the distribution of seeds in the seed bank changes over time.

For each run we simulated population dynamics for

${ }^{1}$ Code that appears in the American Naturalist is provided as a convenience to the readers. It has not necessarily been tested as part of the peer review. 
10,000 time steps and recorded the total population density for the seed bank population: $\|n(\cdot, 10,000)\|_{1}=$ $\int_{0}^{D} n(x, 10,000) d x$. We repeated this process 500 times and calculated the long-term mean,

$$
\text { mean }=\frac{\sum\|n(x, 10,000)\|_{1}}{500},
$$

and variance,

$$
\text { variance }=\sum \frac{\left(\|n(x, 10,000)\|_{1}-\text { mean }\right)^{2}}{500},
$$

of the total seed bank population. Notice that the mean and variance studied here are means and variances across simulations and not across time steps, although ast $\rightarrow \infty$, these two measurements become equivalent.

An IPM's population size can asymptote to zero but never actually reach zero. Thus, we define a quasi-extinction threshold (the minimum viable population density) to be $\|n(\cdot, t, \omega)\|_{1}=50$ seeds (area $)^{-1}$, and the quasiextinction probability as the proportion of 100 simulations with initial population density $\|n(\cdot, 0)\|_{1}=500$ seeds $(\text { area })^{-1}$ that drop below 50 seeds $(\text { area })^{-1}$ at $t=1,000$. The exact value of the quasi-extinction threshold did not influence the qualitative predictions of the model. We repeated these calculations for 90 evenly spaced $(h, \rho)$ combinations in $[0,1] \times[0,1]$ to explore the effect of the probability of disturbance $h$ and the mean depth of disturbance $\rho$ on the mean and variance of the seed bank size and the quasi-extinction probability.

\section{Results}

\section{Projections of Seed Population Dynamics}

Our initial exploratory simulations using various parameter combinations suggest that the probability of disturbance $h$ and fecundity $c$ have a crucial impact on population dynamics. Figure 1 illustrates four typical population simulations and the trajectories of the associated mean-field model. To construct the mean-field model we took the arithmetic average of all possible disturbance kernels and used that kernel as the disturbance kernel every time step, using the probability of disturbance $h$ and the mean depth of disturbance $\rho$ to weight the likelihood of each disturbance kernel. This was done to mimic the deterministic (although sometimes periodic) models used in other articles (Doyle et al. 1986; McGraw 1986; Jordan et al. 1995; GonzalezAndujar 1997; Mertens et al. 2002; Pekrun 2005; Mohler 2006; van den Berg et al. 2010). Low disturbance frequency $h$ and low fecundity $c$ always result in quasi-extinction within $\sim 1,000$ years (fig. 1a). Seeds require a disturbance to germinate; thus, in the periods between disturbances, seed density decreases (due to seed mortality). If the time between disturbances is too long and seed production following a disturbance is too low to offset seed losses due to mortality, the population eventually becomes extinct. In contrast, the mean-field model produces a globally stable, persistent population of $\sim 3,000$ seeds $(\text { area })^{-1}$. Increasing $c$ while keeping the $h$ low produces boom-or-bust dynamics and delayed population extinction for the stochastic model (fig. $1 b, 1 c$ ). Plants with high fecundity contribute many new seeds into the seed bank each time there is a disturbance. Thus, seed density is generally sufficiently high enough for populations to persist for quite some time, even with long intervals between disturbances (the mean time between disturbances is a decreasing function of $h$ ). However, when simulating populations over longer time periods, the seed density eventually decreases below the quasiextinction threshold, due to the nonzero probability of a prolonged streak of years with no disturbance for small $h$. In contrast, the analogous mean-field model predicts high equilibrium seed population sizes $(\sim 5,000$ and 6,000 seeds $\left.(\text { area })^{-1}\right)$. High $c$ combined with increased $h$ produces fluctuations well above the quasi-extinction threshold (but generally below the equilibrium of the mean-field model), even if we extend the simulation interval past the 1,000 time steps shown (fig. 1d).

\section{Long-Term Mean and Variance of Seed Bank Size}

The total seed bank population size is positively correlated with disturbance frequency, $h$, and fecundity $c$ (figs. 2, S2). Reducing density dependence (higher $\alpha$ values) and increasing maximum germination probability $g_{0}$ also increases seed bank size (figs. S3, S4). When maximum seed survival $s_{0}$ is low (fig. $2 a, 2 b$ ) the effect of the mean depth of disturbance $\rho$ depends on the disturbance probability $h$. If disturbance frequency is low, increasing disturbance depth reduces seed bank population size, but disturbance depth has the opposite effect if disturbance frequency is high. This is because, as $\rho$ increases, more newly created seeds are being brought from the surface of the soil to deeper layers where germination rarely happens, and seeds are likely to die before getting back to soil layers (via disturbance) where germination is more likely. In contrast, when maximum seed survival is high, deeper soil layers act as safe sites, and hence, increasing mean depth of disturbance always increases population size, regardless of $h$ (figs. $2 c, 2 d, \mathrm{~S} 2$ ). In this case deep soil layers produce a storage effect; that is, they provide a way of buffering against the effects of years without disturbance. Seed survival as a function of seed depth is also influenced by the rate parameter $b$ (see eq. [4]), and we can use this parameter to explore the role of seed survival close to the surface in producing the nonmonotonic effect of $\rho$ on seed bank size. Seed survival is similar close to the surface for $s_{0}=0.5, b=10$ and for $s_{0}=0.95, b=5$ (fig. S5), 
(a)

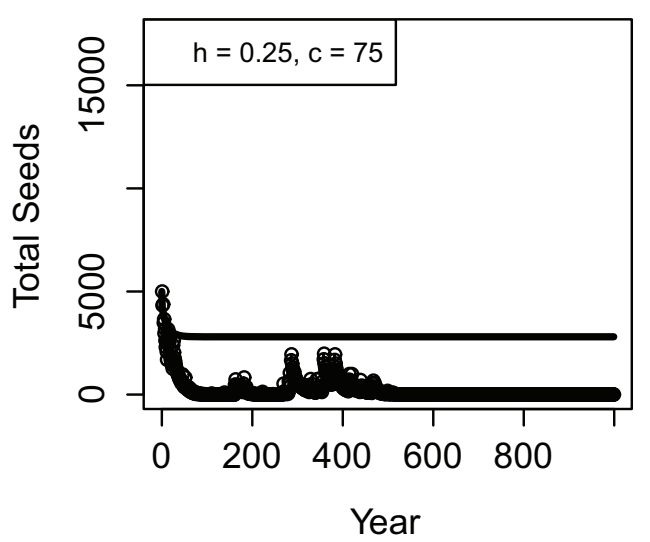

(c)

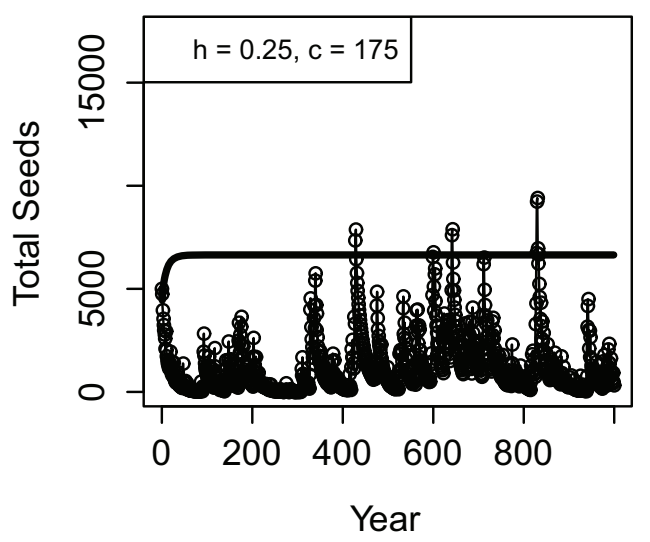

(b)

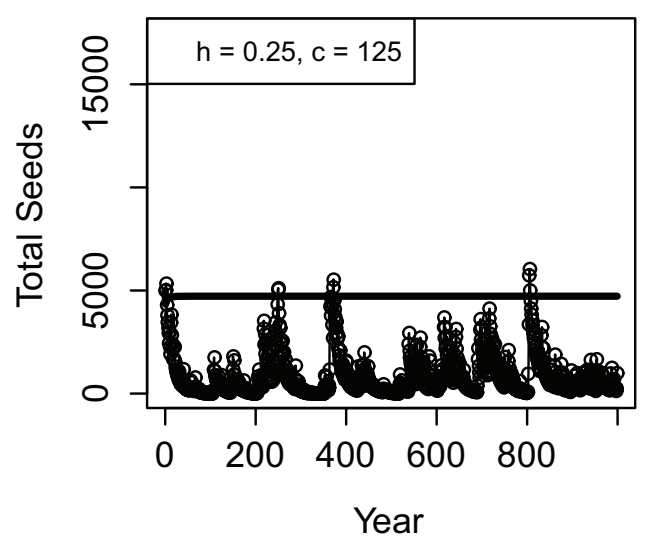

(d)

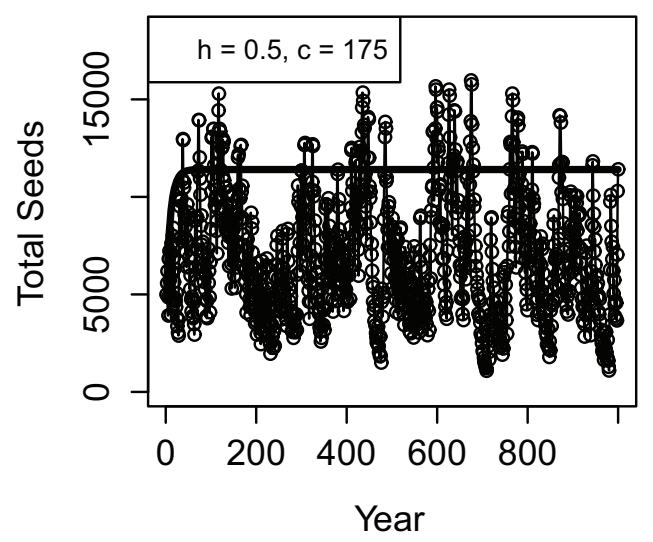

Figure 1: Example simulations of the total seed bank population for four scenarios with different probability of disturbance $h$ and fecundity $c$. The mean depth of disturbance $\rho=0.5$ and the Holling parameters $\alpha=40, \beta=50$. The thick black line is the trajectory of the mean field model associated with each stochastic model. The disturbance kernels in each mean field model are the same each time step and are the arithmetical average of the disturbance kernels used in each stochastic model.

but in one case $\rho$ has a monotonic effect on seed bank population size, and in the other case the effect is nonmonotonic (cf. fig. $2 a, 2 b$ with fig. S $c$, S2d), suggesting that the presence or absence of a storage effect determines whether a small disturbance depth $\rho$ can have a beneficial effect on seed bank population size.

The variance of seed bank size is significantly influenced by the disturbance probability $h$ and has a roughly parabolic shape for most mean depths of disturbance, $\rho$ (fig. 3 ). The initial increase of the variance with increasing $h$ is consistent with an increasing mean seed bank size because the variance typically increases with the mean (fig. 2 ). However, when $h$ is sufficiently large, the carrying capacity of the population imposes an upper bound to the number of seeds that could be produced within one time step. This upper bound reduces the variance in seed production because the population can only have large fluctuations toward the zero population.

Changes in the other model parameters $\left(c, \alpha, \beta, g_{0}, a\right)$ influence the minimum disturbance frequency required for population persistence, but they do not affect the qualitative model predictions (whether there is a nonmonotonic relationship between this long-term mean and $\rho$; figs. 2, S2-S4).

\section{Quasi-Extinction Probability}

Increasing the probability of disturbance $h$ decreases the quasi-extinction probability, regardless of $\rho$ (fig. 4). Seeds need disturbances to germinate, so increasing the disturbance frequency increases the total seed bank population 
(a) - low fecundity/low seed survival

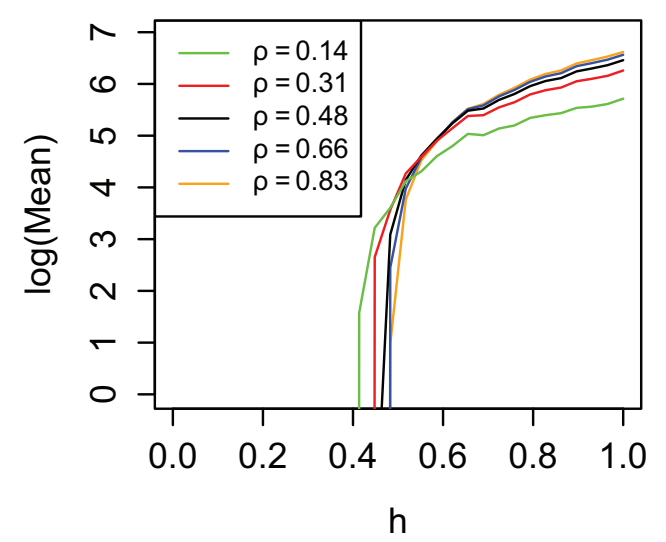

(c) - low fecundity/high seed survival

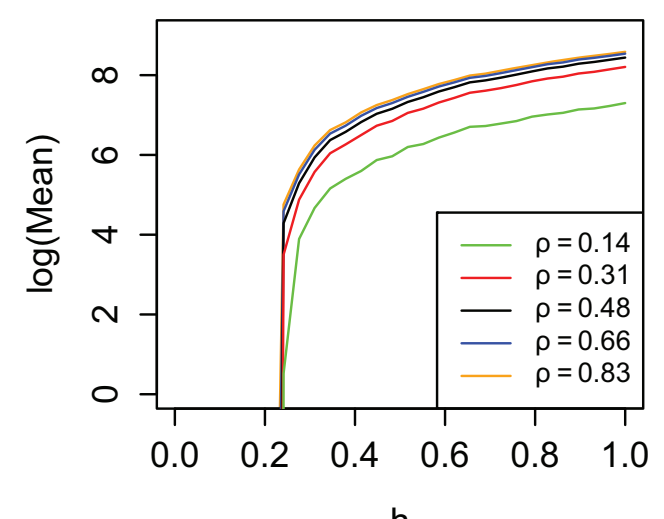

$\mathrm{h}$

\section{(b) - high fecundity/low seed survival}

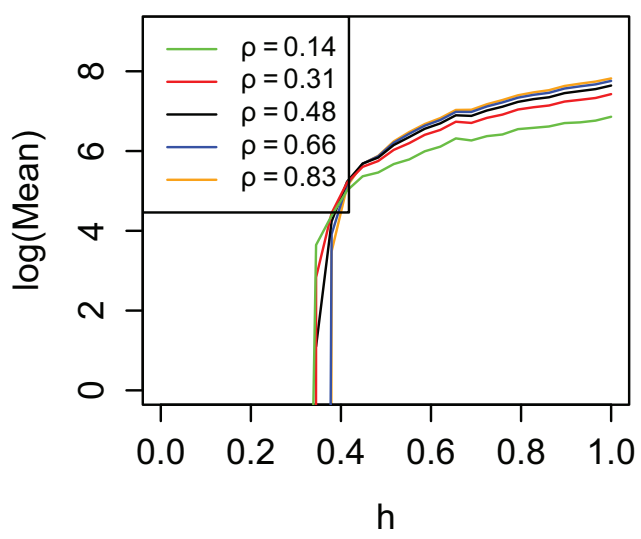

(d) - high fecundity/high seed survival

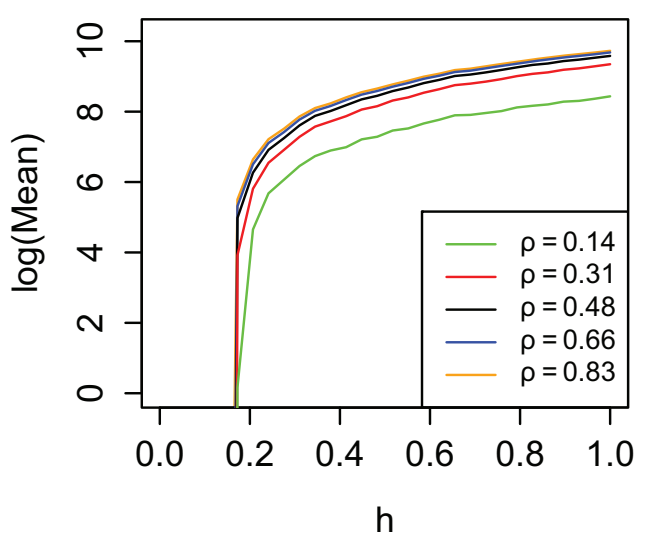

Figure 2: The natural logarithm of the mean of the total seed bank population as a function of the probability of disturbance $h$, with varying mean depth of disturbance $\rho$ after 10,000 time steps. In all graphs $\alpha=40, \beta=50, g_{0}=0.95, a=10$, and $b=10$. $a, c=50$, $s_{0}=0.5 ; b, c=150, s_{0}=0.5 ; c, c=50, s_{0}=0.95 ; d, c=150, s_{0}=0.95$.

(fig. 2), which reduces the extinction probability. The higher the fecundity $c$, the lower the required $h$ needed to prevent extinction (fig. $4 b, 4 d$ ). Interestingly, in some cases increasing the mean depth of disturbance, $\rho$, actually increases the quasi-extinction probability (fig. $4 a, 4 b)$. This happens whenever increasing $\rho$ decreases mean seed bank population size (i.e., in the absence of a storage effect).

\section{Discussion}

The goal of our model was to isolate the effects of random disturbances on the long-term, stochastic equilibrium population dynamics of disturbance specialist plant species in natural environments. Explicitly incorporating the redistribution of seeds in the seed bank is an important part of this work because seed survival and germination are determined by seed depth in many systems (Mohler 1993; Jordan et al. 1995; Gonzalez-Andujar 1997; Mohler et al. 2006; Colbach et al. 2008). The particular shapes describing how seed survival and germination varies with seed depth in our model are applicable to a variety of species (Mohler 1993; Colbach et al. 2008). Our model shows that population persistence is an increasing function of disturbance frequency, which is consistent with previous empirical work studying the dynamics of disturbance specialist plants (Moody-Weis and Alexander 2003; Alexander et al. 2009) and some modeling work studying genetically modified plants (Claessen et al. 2005a, 2005b) and weed seeds (Doyle et al. 1986; Gruber et al. 2004).

The novel insight from this work is that there is a tradeoff between missing germination opportunities and storage effect. When disturbances are rare, but deep, there is 
(a) - low fecundity/low seed survival

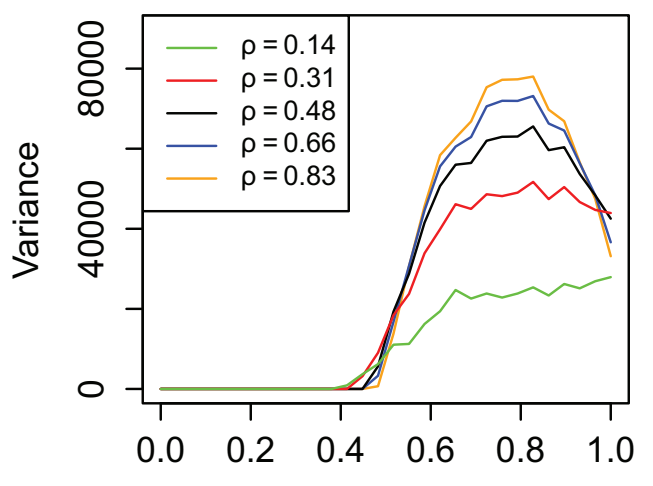

$\mathrm{h}$

(c) - low fecundity/high seed survival

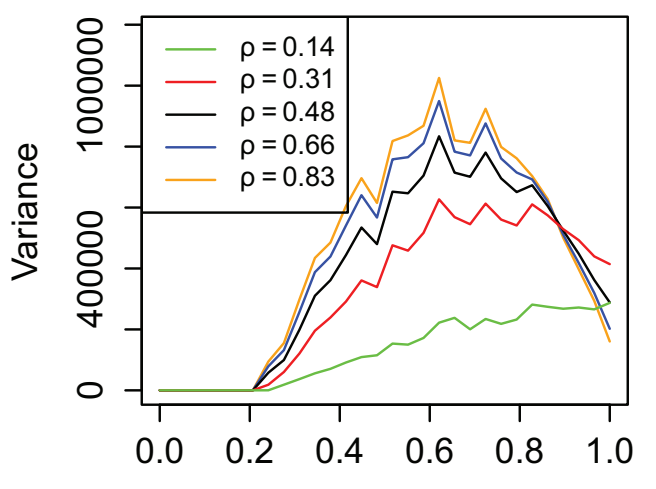

$\mathrm{h}$

\section{(b) - high fecundity/low seed survival}

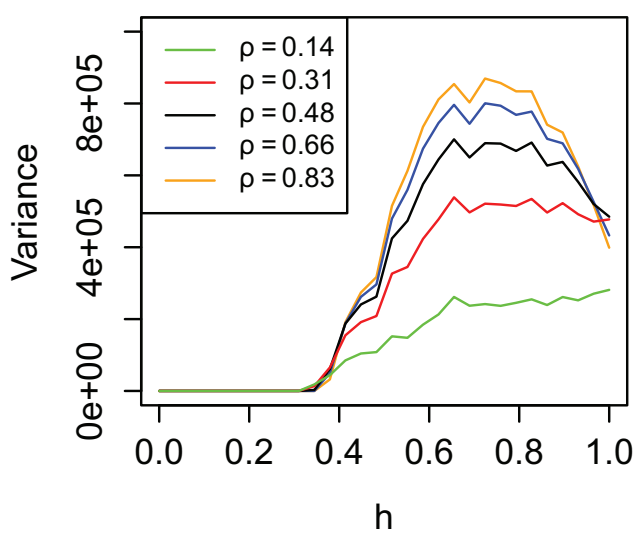

(d) - high fecundity/high seed survival

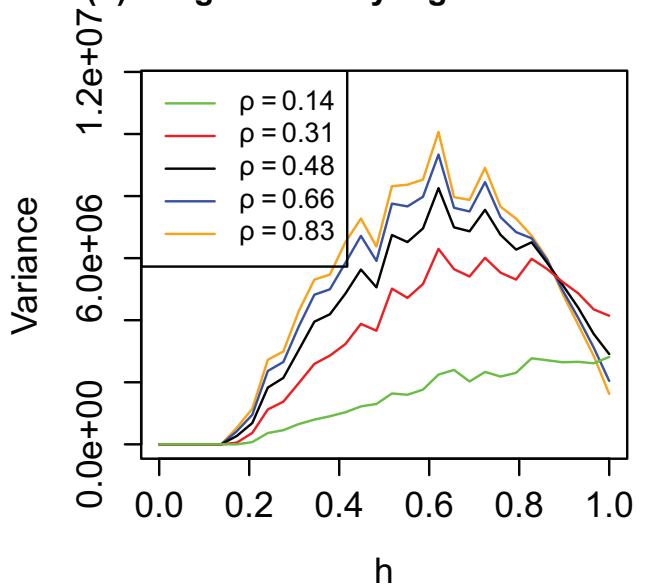

Figure 3: The variance of the total seed bank population as a function of the probability of disturbance $h$, with varying mean depth of disturbance $\rho$ after 10,000 time steps. The parameter values in this figure are the same as those in figure 2 .

a high probability of seeds being moved to deeper depths, where the germination rate is very low. If seed survival in these deep soil layers is low (e.g., the thistle species Cirsium vulgare; Tenhumberg et al. 2008), then a seed has a high probably of dying before it has a chance to reach the surface of the soil again to germinate. Thus, shallow disturbances are more advantageous for seeds because they allow them to stay close to the surface and not miss any germination opportunity. This suggests that in environments where a population is near the quasi-extinction boundary, deep disturbances can decrease population viability because the dynamics when the population is small often dictates the persistence or extinction of the population (Hardin et al. 1988; Benaim and Schreiber 2009). In contrast, when seed survival in deep layers is high (safe sites; e.g., the wild sunflower species Helianthus annuus;
Alexander and Schrag 2003; Alexander et al. 2009), seeds have a high a chance of surviving until the next time they are brought to the surface of the soil via a disturbance, even though these events are infrequent. Thus, deep disturbances are beneficial for plants because they move seeds to safe sites and thereby buffer the population against the negative effects of long periods without germination opportunity (no disturbance). On the other hand, when disturbances are frequent there is always a relatively large number of seeds on the surface ready to germinate (maximum recruitment is limited by density dependence); hence, even a weak storage effect $\left(s_{0}=0.5\right)$ is beneficial.

To our knowledge, this article is the first modeling study evaluating the effect of average depth of disturbance on population viability. Many models for weed seeds have considered depth as a stage variable for the seed bank 
(a) - low fecundity/low seed survival

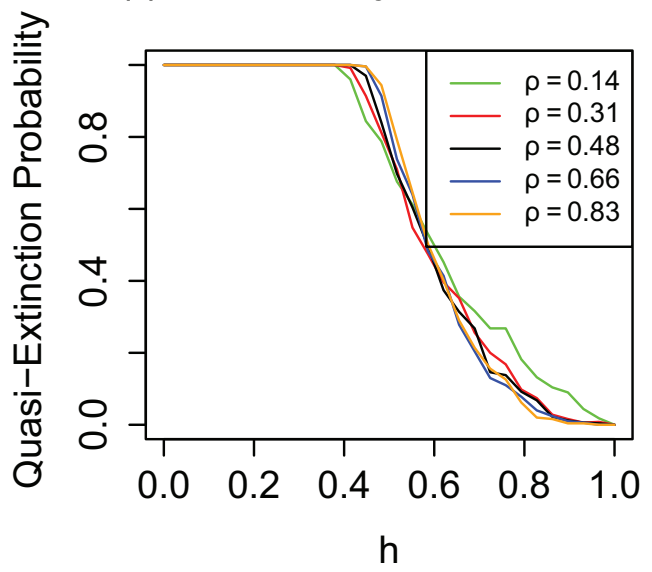

(c) - low fecundity/high seed survival

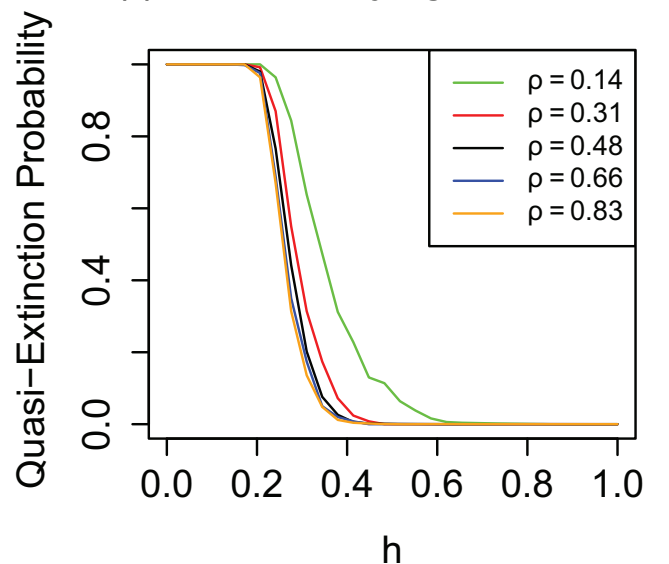

(b) - high fecundity/low seed survival

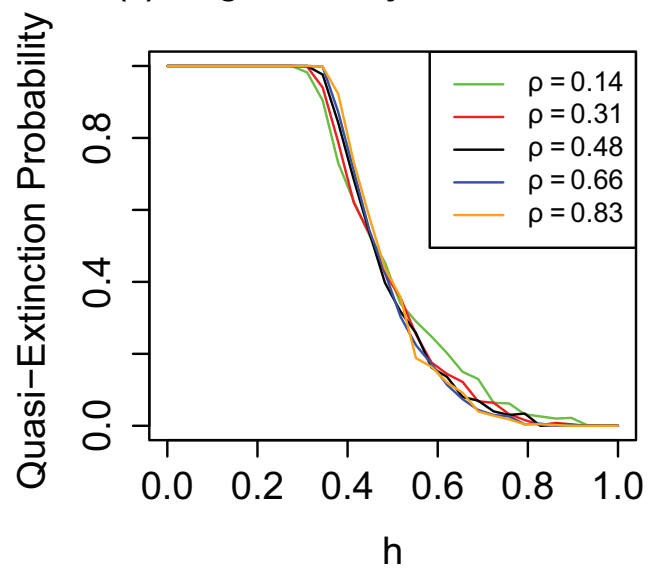

(d) - high fecundity/high seed survival

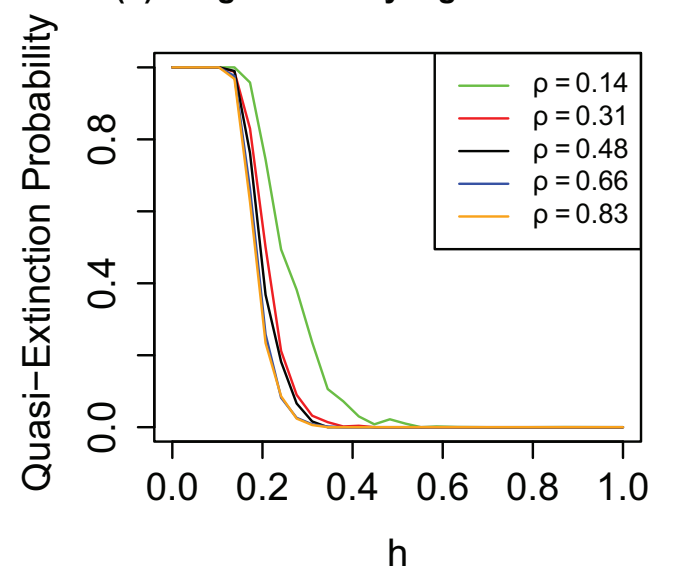

Figure 4: Probability of dropping below the quasi-extinction threshold of 50 seeds/area by time $t=1,000$, as a function of the probability of disturbance $h$, with varying mean depth of disturbance $\rho$ after 10,000 time steps. The parameter values in this figure are the same as those in figure 2.

(Froud-Williams et al. 1984; Doyle et al. 1986; McGraw 1986; Mohler 1993; Jordan et al. 1995; Gonzalez-Andujar 1997; Mertens et al. 2002; Claessen et al. 2005a, 2005b; Pekrun et al. 2005; Mohler et al. 2006; van den Berg et al. 2010), but instead of understanding the effect of average depth of disturbance on stochastic population dynamics, these studies have focused on how the yield of agricultural crops is influenced by different management strategies (i.e., crop rotation) and tilling regimes. For example, Claessen et al. (2005a) explored how changes in model parameters would affect the population growth rate (sensitivity analysis), but the effect of the transition probabilities between the top and bottom layers in the soil was not included in the analysis. Mertens et al. (2002) and van den Berg et al. (2010) perform sensitivity and elasticity analysis of weed population growth rate for Polygonum persicaria and Persicaria maculosa, respectively, but their models are deterministic or periodic, not stochastic.

In natural environments, the maximum disturbance depth is likely to vary between different plant species, and the spatiotemporal variation in abiotic and biotic factors is likely to produce variation in mean disturbance depths. For instance, soil characteristics and precipitation are likely to influence disturbance depth because in dry loamy soil it is more challenging for small mammals to dig deep tunnels, and birds might prefer to cache seeds at a shallower depth than they would choose in moister or sandier soil. Furthermore, variation in the distribution and composition of disturbance agents will influence how deeply seeds are buried in the soil (relative to the maximum depth for a specific plant species). Anthropogenic factors might be important too, as they influence the fire frequency and 
grazing intensity (shallow disturbances). Untangling empirically the interactions between the nature of local disturbance events and the plant's response to disturbances (Sousa 1984) is costly, but the results of our model suggest that these interactions may partially explain spatiotemporal variation in species persistence.

Previous models were concerned with evaluating the outcomes of different tilling regimes as weed management strategies, using deterministic sequences of disturbances (Doyle et al. 1986; McGraw 1986; Maxwell 1988; Mohler 1993; Jordan et al. 1995; Gonzalez-Andujar 1997; Mertens et al. 2002; Pekrun et al. 2005; Garnier and Lecomte 2006; Sester et al. 2007; van den Berg et al. 2010). In contrast, we model disturbances as a stochastic process mimicking disturbance sequences in natural environments. We show in this article that the predictions of a stochastic model can be dramatically different from those of a model incorporating the average effect of disturbances (fig. 1a population extinction vs. equilibrium seed population size of $\sim 3,000$ seeds $\left.(\text { area })^{-1}\right)$. It is well known that deterministic models typically overestimate population viability (Tuljapurkar 1990; Caswell 2001), however, rarely is the predicted difference this large. The enhanced difference that we see is likely due to the presence of density dependence in our model. The nonlinear function $f$ modeling seedling recruitment is concave down in our model (see eq. [7]), and thus, by Jensen's inequality, the second part of equation (8) yields the inequality

$$
\begin{aligned}
\mathrm{E}[p(t+1)]=\mathrm{E}\left\{f \left[\int_{0}^{D} g(x, \theta(t)) \int_{0}^{D} K(x, y, \theta)\right.\right. \\
\times(n(y, t)+J(y) p(t)) d y d x]\} \\
\leq f\left\{\int_{0}^{D} \mathrm{E}[g(x, \theta(t))] \int_{0}^{D} \mathrm{E}[K(x, y, \theta)]\right. \\
\times[\mathrm{E}(n(y, t))+J(y) \mathrm{E}(p(t))] d y d x\},
\end{aligned}
$$

where $\mathrm{E}$ is the expected value (mean) with respect to the stochastic process $\theta(t)$. The first term refers to the mean of the long-term population in our article, while the second is the mean field object usually assumed by previous work for agricultural models.

\section{Conclusion}

We developed an integral projection model that mechanistically incorporates the stochastic effect of random, nat- ural disturbances on population dynamics of disturbance specialist plants by explicitly considering the continuous vertical dynamics of seeds in the seed bank and density dependence in seedling recruitment. Our model suggests that frequency and intensity of disturbances are important drivers of the population dynamics. Generally, a high disturbance frequency promotes population persistence, but the effect of mean disturbance depth on population viability is surprising. If seed survival is low, deep disturbances can increase extinction risk of already threatened populations by reducing seed population size, while in favorable environments the effect is opposite, and deep disturbances increase seed population size. Furthermore, our model suggests that conclusions from agroecosystems may not always apply to natural systems.

\section{Acknowledgments}

We would like to thank S. Dunbar and S. Reynolds for their important roles in the discussions pertaining to the development of the model in this article. The authors would also like to thank Editor Troy Day, Associate Editor Andy Gardner, and three anonymous reviewers for their helpful comments and suggestions that greatly improved this manuscript.

\section{Literature Cited}

Alexander, H. M., D. Pilson, J. Moody-Weis, and N. A. Slade. 2009. Geographic variation in dynamics of an annual plant with a seed bank. Journal of Ecology 97:1390-1400.

Alexander, H. M., and A. M Schrag. 2003. Role of soil seed banks and newly dispersed seeds in population dynamics of the annual sunflower, Helianthus annuus. Journal of Ecology 91:987-998.

Benaim, M., and S. J. Schreiber. 2009. Persistence of structured populations in random environments. Theoretical Population Biology 76:19-34.

Caswell, H. 2001. Matrix population models: construction, analysis and interpretation. 2nd ed. Springer, New York.

Chancellor, R. J. 1964. Emergence of weed seedlings in the field and the effects of different frequencies of cultivation. Pages 607-613 in Proceedings of the 7th British Weed Control Conference. British Crop Protection Council, London.

Claessen, D., C. A. Gilligan, J. W. Lutman, and F. van den Bosch. $2005 a$. Which traits promote persistence of feral GM crops? pt. 1: implications of environmental stochasticity. Oikos 110:20-29.

Claessen, D., C. A. Gilligan, and F. van den Bosch. 2005b. Which traits promote persistence of feral GM crops? pt. 2: implications of metapopulation structure. Oikos 110:30-42.

Colbach, N., C. Durr, S. Gruber, and C. Pekrun. 2008. Modelling the seed bank evolution and emergence of oilseed rape volunteers for managing co-existence of GM and non-GM varieties. European Journal of Agronomy 28:19-32. 
Coulson, T. 2012. Integral projection models, their construction and use in posing hypotheses in ecology. Oikos 212:1337-1350.

Doyle, C. J. 1991. Mathematical models in weed management. Crop Protection 10:432-444.

Doyle, C. J., R. Cousens, S. R. Moss. 1986. A model of the economics of controlling Alopecurus myosuroides Huds. in winter wheat. Crop Protection 5:143-150.

Eager, E. A., R. Rebarber, and B. Tenhumberg. 2012. Choice of density dependent seedling recruitment function affects predicted transient dynamics: a case study with Platte thistle. Theoretical Ecology 5:387-401.

- 2013. Global asymptotic stability of plant-seed bank models. Journal of Mathematical Biology, doi:10.1007/s00285-013-0689-z.

Easterling, M. R., S. P. Ellner, and P. M. Dixon. 2000. Size-specific sensitivity: applying a new structured population model. Ecology 81:694-708.

Edelstein-Keshet, L. 2005. Mathematical models in biology. Society for Industrial and Applied Mathematics, Philadelphia.

Ellner, S. P., and J. Guckenheimer. 2006. Dynamic models in biology. Princeton University Press, Princeton, NJ.

Ellner, S. P., and M. Rees. 2006. Integral projection models for species with complex demography. American Naturalist 167:410-428.

. 2007. Stochastic stable population growth in integral projection models: theory and application. Journal of Mathematical Biology 54:227-256.

Fenner, M., and K. Thompson. 2005. The ecology of seeds. Cambridge University Press, Cambridge.

Froud-Williams, R. J., R. J. Chancellor, and D. S. H. Drennan. 1984. The effects of seed burial and soil disturbance on emergence and survival of arable weeds in relation to minimal cultivation. Journal of Applied Ecology 21:629-641.

Garnier, A., A. Deville, and J. Lecomte. 2006. Stochastic modeling of feral plant populations with seed immigration and road verge management. Ecological Modelling 197:373-382.

Garnier, A., and J. Lecomte. 2006. Using a spatial and stage-structured invasion model to assess the spread of feral populations of transgenic oilseed rape. Ecological Modelling 194:141-149.

Gonzalez-Andujar, J. L. 1997. A matrix model for the population dynamics and vertical distribution of weed seedbanks. Ecological Modelling 97:117-120.

Gruber, S., C. Pekrun, and W. Claupein. 2004. Population dynamics of volunteer oilseed rape (Brassica napus L.) affected by tillage. European Journal of Agronomy 20:351-361.

Hardin, D. P., P. Takac, and G. F. Webb. 1988. Asymptotic properties of a continuous-space discrete-time population model in a random environment. Journal of Mathematical Biology 26:361-374.

Holling, C. S. 1959. Some characteristics of simple types of predation and parasitism. Canadian Entomologist 91:385-398.

Jordan, N., D. A. Mortensen, D. M. Prenzlow, and K. C. Cox. 1995. Analysis of crop rotation on weed seedbanks. American Journal of Botany 82:390-398.

Kalisz, S., and M. A. McPeek. 1992. Demography of an age-structured annual: resampled projection matrices, elasticity analyses, and seed bank effects. Ecology 73:1082-1093.
MacDonald, N., and A. R. Watkinson. 1981. Models of an annual population with a seedbank. Journal of Theoretical Biology 93:643653.

Maxwell, B. D., M. V. Wilson, and S. R. Radosevich. 1988. Population modeling approach for evaluating leafy spurge (Euphorbia esula) development and control. Weed Technology 2:132-138.

McGraw, J. B. 1986. Seed-bank properties of an Appalachian sphagnum bog and a model of the depth distribution of viable seeds. Canadian Journal of Botany 65:2028-2035.

Mertens, S. K., F. van den Bosch, and J. A. P. Heesterbeek. 2002. Weed populations and crop rotations: exploring dynamics of a structured periodic system. Ecological Applications 12:1125-1141.

Miller, A. D., S. H. Roxburgh, and K. Shea. 2011. Timing of disturbance alters competitive outcomes and mechanisms of coexistence in an annual plant model. Theoretical Ecology 5:419-432, doi: 10.1007/s12080-011-0133-1.

Mohler, C. L. 1993. A model of the effects of tillage on emergence of weed seedlings. Ecological Applications 3:53-73.

Mohler, C. L., J. C. Frisch, and C. E. McCulloch. 2006. Vertical movement of weed seed surrogates by tillage implements and natural processes. Soil and Tillage Research 86:110-122.

Moody-Weis, J., and H. M. Alexander. 2007. The mechanisms and consequences of seed bank formation in wild sunflowers (Helianthus annuus). Journal of Ecology 95:851-864.

Pekrun, C., P. W. Lane, and P. J. W. Lutman. 2005. Modelling seedbank dynamics of volunteer oilseed rape (Brassica napus). Agricultural Systems 84:1-20.

R Development Core Team. 2006. R: a language and environment for statistical computing. Vienna, Austria.

Roberts, H. A. 1981. Seed banks in soils. Advances in Applied Biology $6: 1-55$.

Sester, M., C. Durr, H. Darmency, and N. Colbach. 2007. Modelling the effects of cropping systems on the seed bank dynamics and the emergence of weed beet. Ecological Modelling 204:47-58.

Sousa, W. P. 1984. The role of disturbance in natural communities. Annual Review of Ecology and Systematics 15:353-391.

Tenhumberg, B., S. M. Louda, J. O. Eckberg, and M. Takahashi. 2008. Monte Carlo analysis of a parameter uncertainty in matrix models for the weed Cirsium vulgare. Journal of Applied Ecology 45:438447.

Tenhumberg, B., T. J. Tyre, and R. Rebarber. 2009. Model complexity affects transient population dynamics following a dispersal event: a case study with pea aphids. Ecology 90:1878-1890.

Tuljapurkar, S. 1990. Population dynamics in variable environments. Lecture Notes in Biomathematics. Springer, Berlin.

van den Berg, F., C. A. Gilligan, J. C. Gerdessen, L. A. H. Gregoire, and F. van den Bosch. 2010. Optimal weed management in crop rotations: incorporating economics is crucial. Weed Research 50: 413-424.

Venable, D. L. 1989. Modeling the evolutionary ecology of seed banks. Pages 67-87 in M. A. Leck, V. T. Parker, and R. L. Simpson, eds. Ecology of seed banks. Academic Press, Waltham, MA.

Associate Editor: Andy Gardner Editor: Troy Day 